\title{
Gabies - campagne de 2020 du musée du Louvre
}

\section{Steve Glisoni}

\section{(2) OpenEdition \\ Journals}

Édition électronique

URL : https://journals.openedition.org/baefe/4359

DOI : $10.4000 /$ baefe.4359

ISSN : 2732-687X

Éditeur

ResEFE

\section{Référence électronique}

Steve Glisoni, « Gabies - campagne de 2020 du musée du Louvre » [notice archéologique], Bulletin archéologique des Écoles françaises à l'étranger [En ligne], Italie, mis en ligne le 29 septembre 2021, consulté le 02 octobre 2021. URL : http://journals.openedition.org/baefe/4359; DOI : https://doi.org/ 10.4000/baefe.4359

Ce document a été généré automatiquement le 2 octobre 2021.

\section{cc) $(1) \odot$}

Le Bulletin archéologique des Écoles françaises à l'étranger est mise à disposition selon les termes de la Licence Creative Commons Attribution - Pas d'Utilisation Commerciale - Pas de Modification 4.0 International. 


\title{
Gabies - campagne de $2020 \mathrm{du}$ musée du Louvre
}

\author{
Steve Glisoni
}

\section{NOTE DE L'AUTEUR}

Date précise de l'opération : 5 au 30 octobre 2020

Autorité nationale présente : Soprintendenza Speciale Archeologia Belle Arti e Paesaggio di Roma

Composition de l'équipe de terrain : Steve Glisoni (archéologue, Inrap, musée du Louvre), Isabelle Hasselin (conservatrice, musée du Louvre), Florence Specque (documentaliste scientifique, musée du Louvre), Carlo Monda (architecte - responsable de la sécurité du chantier), Mehdi Belarbi (archéologue - topographe, Inrap), Cristina Molari (céramologue), Nadia Cipolli (céramologue), Sébastien Lepetz (archéozoologue, CNRS, MNHN) Sophie Barat (archéologue, spécialiste du mobilier en verre), Arnaud Coutelas (archéologue, spécialiste des mortiers), Johann Blanchard (archéologue, Inrap), Camille Colonna (archéologue - anthropologue, Inrap), Priscillia Debouige (archéologue, DHAAP/ville de Paris), Gwenaëlle Desforges (archéologue, Inrap), Laura Favreau (Gestionnaire des bases de données, musée du Louvre), Yannick Fouvez (archéologue, Inrap), Lise Mész (archéologue - ville de Paris), Agnès Scherer (documentaliste scientifique, musée du Louvre), Antoine Valois (archéologue, Inrap), Marjolaine Benaich (épigraphiste, doctorante en archéologie, Sorbonne Université Paris IV), Heidelinde Hupfer (doctorante en archéologie, École du Louvre), Élise Mousson (étudiante en archéologie, Faculté des Sciences historiques, Université de Strasbourg), Jordan Voillot (étudiant en archéologie, École Nationale Supérieure d'Architecture de Strasbourg et Faculté des Sciences historiques, Université de Strasbourg), Euan Wall (chargé d'études et de recherche, INHA, doctorant, Université de Paris 1), Janet Lepetz. 


\section{Partenariats institutionnels :}

Soprintendenza Speciale Archeologia Belle Arti e Paesaggio di Roma ( $\mathrm{n}^{\circ}$ de concession MIBACT $n^{\circ} 14409$ du 07/05/2020), Inrap, École française de Rome.

Établissement porteur de l'opération : musée du Louvre

Remerciements : Nous remercions la Surintendance spéciale de Rome et particulièrement la dott.ssa Daniela Porro, Soprintendente Speciale, ainsi que la dott.ssa Chiara Andreotti et le dott. Rocco Bochicchio, responsables du site de Gabies.

Nous remercions vivement la Société de Amis du Louvre pour son soutien à ce projet.

\section{Chroniques de l'EFR :}

Gabies, 2013

Gabies, 2014 et 2016

Gabies, 2017

Gabies, 2018

Gabies, 2019

La mission archéologique du Louvre sur le site de Gabies a réalisé sa septième campagne de fouilles du 5 au 30 octobre 2020. Les recherches ont tout d'abord porté sur la pars postica de la domus d'époque républicaine et une partie du tablinum a pu être mise au jour, ainsi que la pièce qui le jouxte à l'ouest (fig. 1 et 2 ). Cette dernière est flanquée d'un couloir qui donnait accès à la partie arrière de l'édifice où devait se trouver un espace ouvert, un jardin ou un péristyle.

Fig. 1. Vue générale du chantier de fouille lors de la campagne de 2020.

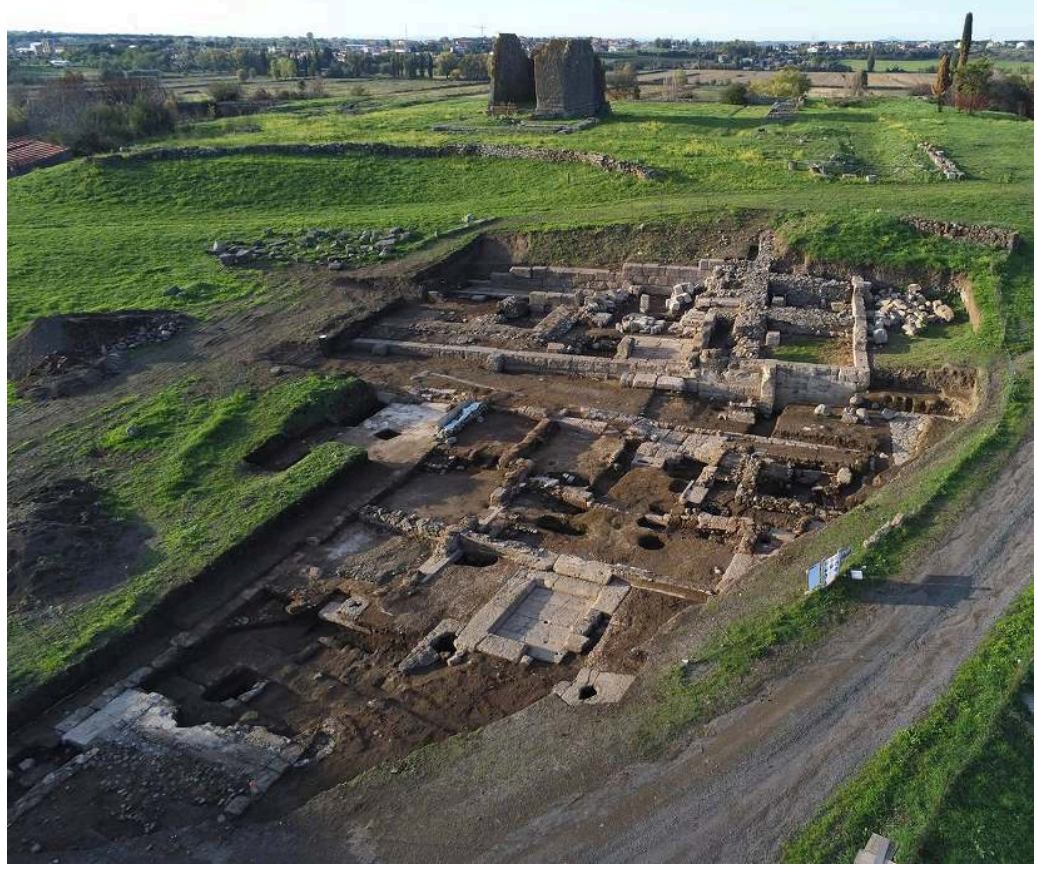

Mehdi Belarbi. 
Fig. 2. Plan général des vestiges (2013-2020).

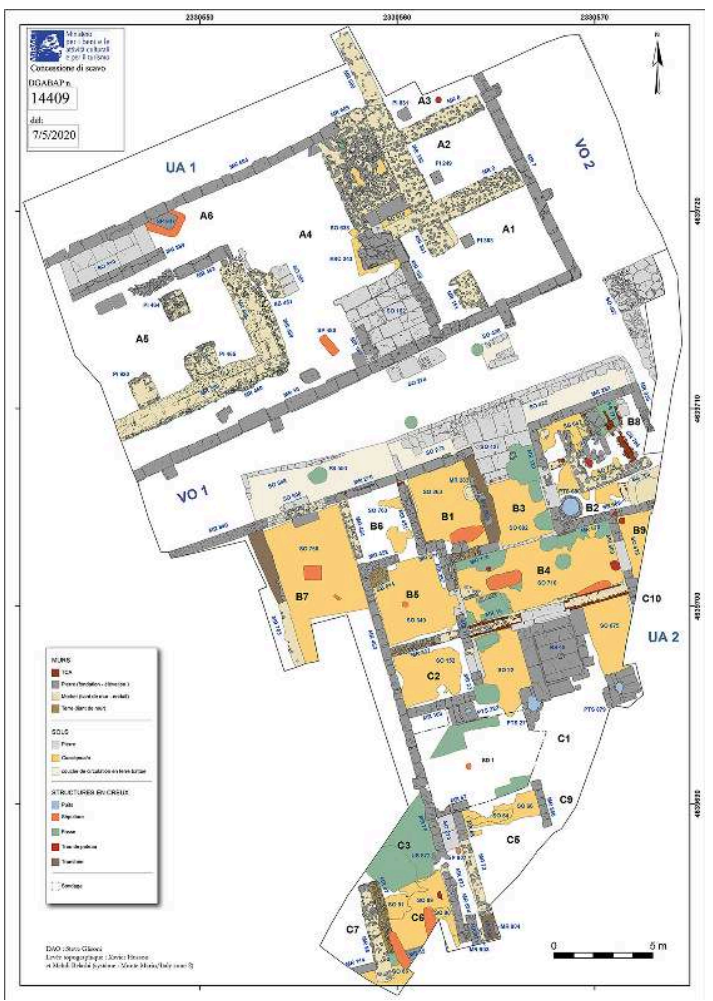

2 Des niveaux d'occupation d'époque archaïque ont également été mis au jour dans ce secteur, ainsi qu'une inhumation d'un périnatal dans une urne funéraire en céramique datée du début du $v^{\mathrm{e}}$ s. av. J.-C.

3 L'extension de l'emprise de fouille vers l'ouest dans le secteur du théâtre du sanctuaire de Junon Gabina a par ailleurs permis de retrouver la base du mur d'estrade, la frons pulpiti, ainsi que le sol dallé qui conduisait à l'orchestra.

\section{Les périodes orientalisante et archaïque}

4 Plusieurs niveaux datés entre le $\mathrm{VII}^{\mathrm{e}}$ et le $\mathrm{VI}^{\mathrm{e}} \mathrm{s}$. av. J.-C. ont été fouillés au sud, dans le secteur $C$ sous des murs et des niveaux de remblais associés à la domus UA 2. Un niveau d'occupation mesurant entre 0,10 et 0,20 m d'épaisseur (US 915 et US 917 ${ }^{1}$ ) a ainsi été mis au jour dans l'emprise du couloir $C 4$ sous les fondations des murs 72, 871 et 874 à $63,20 \mathrm{~m}$ d'altitude (fig. 2). Il est constitué d'un limon sableux compact et homogène légèrement caillouteux brun foncé (fig. 3). 
Fig. 3. Le niveau d'occupation US 915 vu depuis le nord.

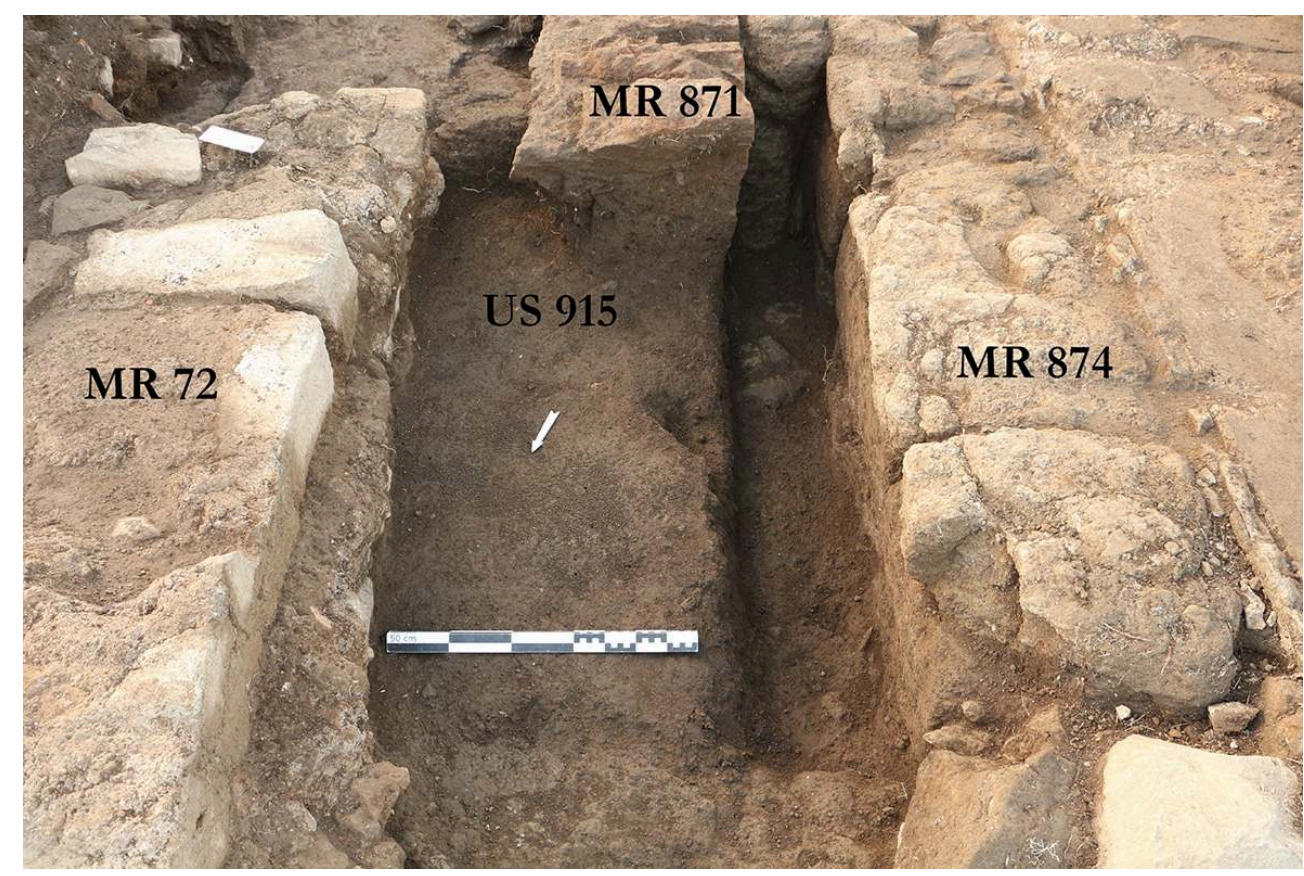

Euan Wall.

5 Le mobilier céramique recueilli dans l'US 917 fournit un teminus post quem daté de la fin du $\mathrm{VII}^{\mathrm{e}}$ s. av. J.-C. et celui de l'US 915, le milieu du $\mathrm{VI}^{\mathrm{e}}$ s. av. J.-C., ce qui place ce niveau d'occupation dans la période archaïque. Le mobilier de l'US 917 est a priori résiduel mais témoigne de la formation possible de cette couche au moins dès la période orientalisante récente. Au sud, sous l'US 915, une séquence de plusieurs couches a été mise en évidence sur près de $0,80 \mathrm{~m}$ d'épaisseur, le banc rocheux étant plus bas à cet endroit. Elles n'ont pas livré de mobilier datant.

Immédiatement au nord, contre le dallage SO 210, a été découverte une sépulture d'un individu périnatal (SP 907) dont les ossements étaient conservés dans une olla déposée dans une fosse sépulcrale (fig. 4). L'olla est datée du début du ve $s$. av. J.-C. La sépulture a été partiellement fouillée en 2020, sa partie nord comprenant une deuxième olla ossuaria se trouvant sous la dalle. Le col des deux olla étaient accolés. L'olla non exhumée contient peut-être d'autres fragments osseux, ce qui expliquerait l'absence d'os longs observés au cours de la fouille. Ce dépôt funéraire s'apparente à un suggrundarium, ce qui pourrait suggérer la présence d'un habitat à proximité immédiate. 
Fig. 4. Olla contenant les ossements d'un individu périnatal.

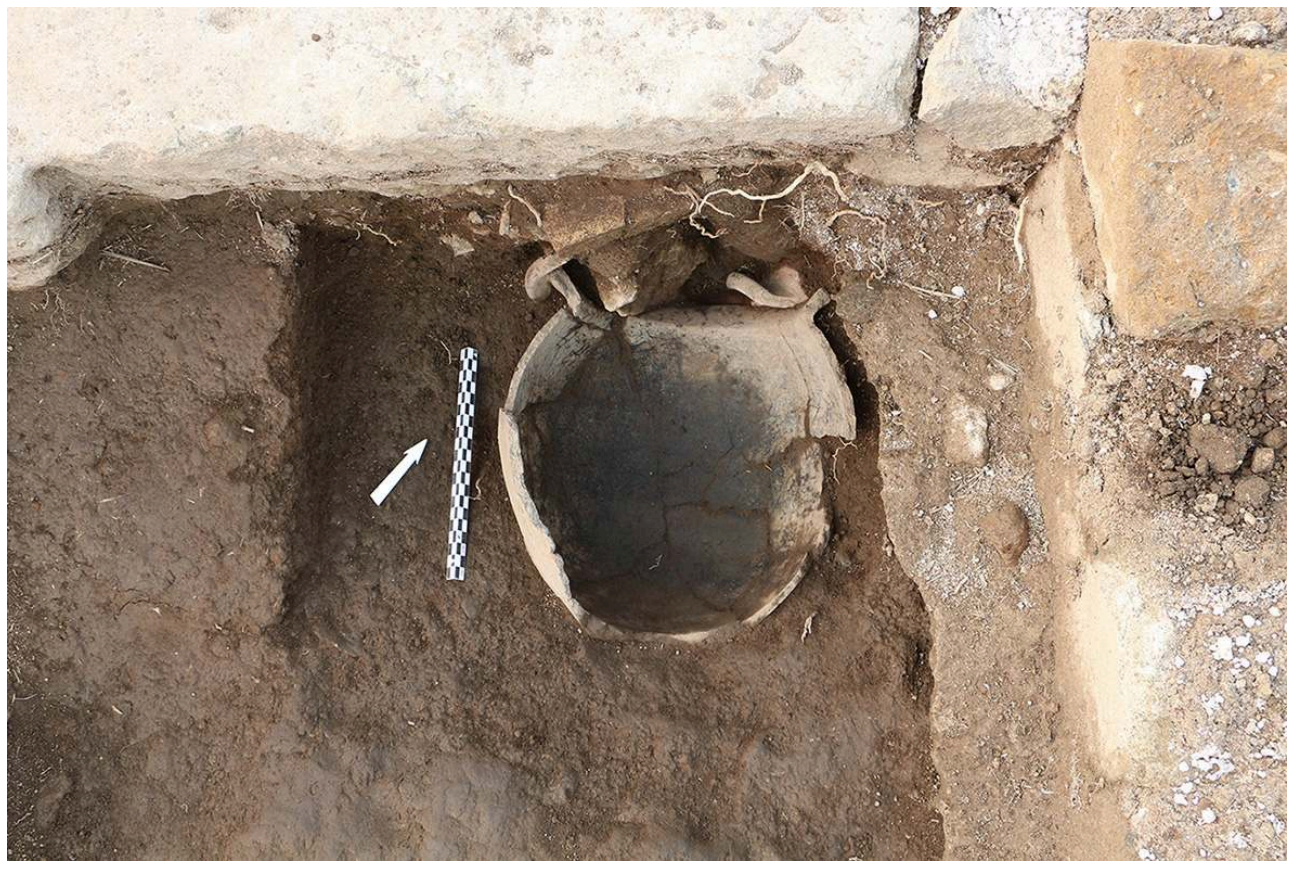

Euan Wall.

$7 \quad$ Une autre sépulture (SP 947) a été découverte à un peu plus d'une trentaine de mètres au nord, sous le mur de soutènement des gradins de la cavea du théâtre du sanctuaire de Junon Gabina (fig. 5).

Fig. 5. Sarcophage d'une sépulture en partie engagée sous le mur de soutènement des gradins de la cavea.

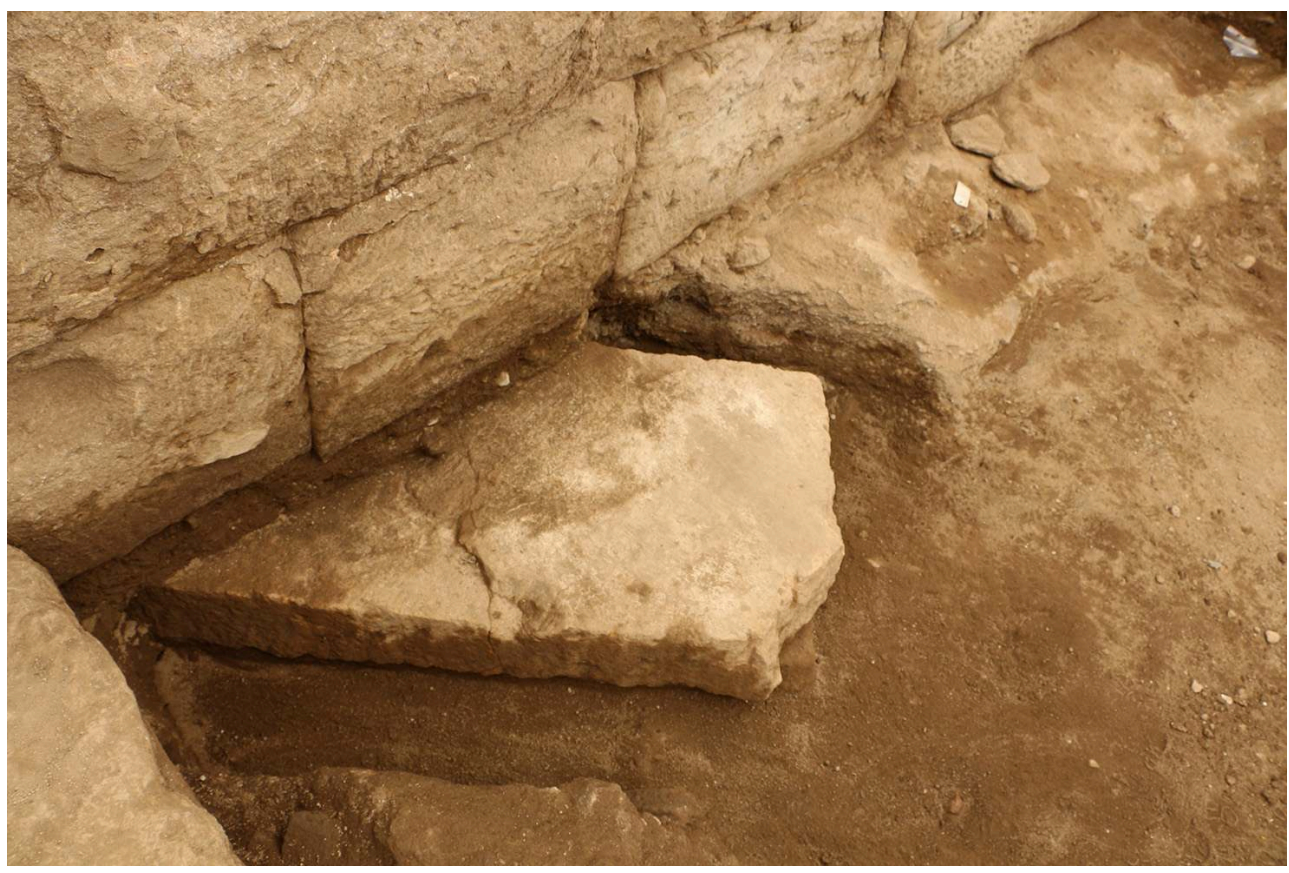

Mehdi Belarbi. 
La partie exhumée comprend une dalle de sarcophage en tuf orangé mesurant 0,78 $\mathrm{m}$ de large et $0,20 \mathrm{~m}$ d'épaisseur qui recouvre un caisson rectangulaire de même nature. Le sarcophage est déposé dans une fosse rectangulaire aux contours arrondis mesurant $1,10 \mathrm{~m}$ de large directement creusée dans le substrat rocheux. En surface, cette sépulture est semblable à la tombe 25 mise au jour dans l'Area $\mathrm{D}$ fouillée par l'équipe du Gabii Project sous la direction de Nicola Terrenato et datée du début du ve s. av. J.-C. ${ }^{2}$

9 Ces sépultures viennent s'ajouter à la sépulture en fosse d'un immature (SP 480) datée de l'époque archaïque fouillée en $2017^{3}$.

\section{La domus UA 2}

10 Le décapage des pièces situées au sud de l'atrium a permis de dégager le tablinum (pièce $\mathrm{C} 9$ ) sur une superficie de $3,80 \mathrm{~m}^{2}$ et la pièce $\mathrm{C} 5$, qui le jouxte à l'ouest sur une superficie de $14,30 \mathrm{~m}^{2}$ (fig. 6). Au total, la domus a été ouverte sur une superficie de $270 \mathrm{~m}^{2}$.

Fig. 6. Vue aérienne zénithale de la pars postica de la domus UA 2 - au centre le sondage 1 Mehdi Belarbi.

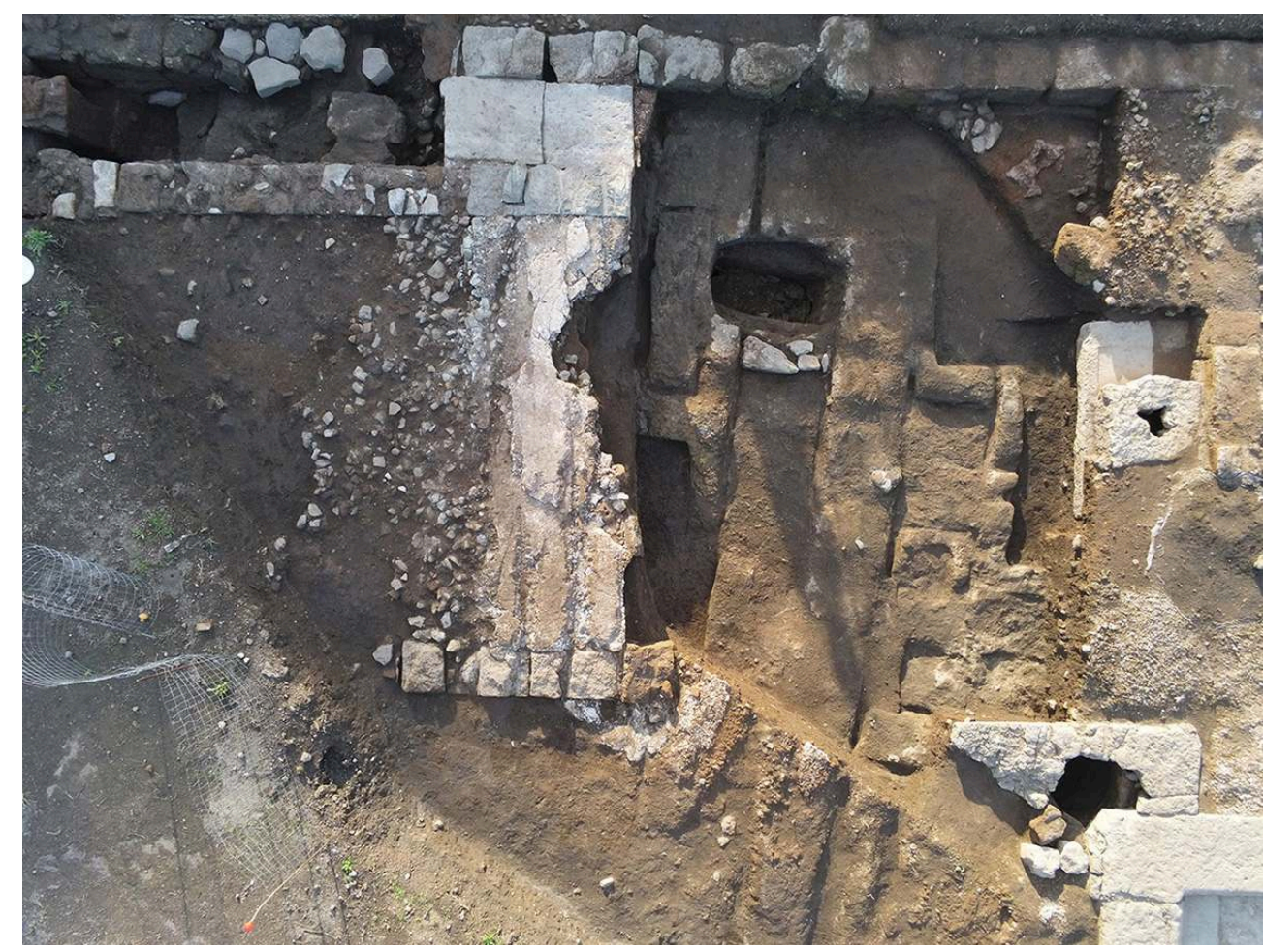

11 Dans l'axe central, le tablinum est complètement ouvert sur l'atrium (C1). Quelques rares lambeaux du radier de sol en béton de la pièce subsistent mais l'essentiel a été détruit par le passage répété de la charrue comme le montrent les sillons nettement visibles laissés par les socs. Le sol en béton de tuileau de la pièce ouest situé à $63,80 \mathrm{~m}$ d'altitude (SO 64 et 66), est mieux conservé dans la partie nord. Il a connu une phase de réfection puisque deux chapes sont visibles l'une sur l'autre. La surface des sols est décorée d'incrustations de tesselles dessinant un semis irrégulier. 
12 partie du radier a permis d'étendre le sondage 1 vers le nord et de fouiller ainsi les remblais de construction du bâtiment qui ont livré du mobilier céramique dont les éléments les plus récents sont produits à partir du milieu du $\mathrm{III}^{\mathrm{e}} \mathrm{s}$. av.J.-C., ce qui confirme et affine la chronologie déjà établie.

13 différentes (fig. 7). Le mur oriental, MR 72, est monté au mortier tandis que le mur occidental, MR 874, est en appareil rectangulaire de lapis Gabinus tout comme le mur MR 70, dans le même alignement plus au nord. Entre ces deux tronçons de mur se placent un bloc de tuf orangé (US 872) en position débordante vers l'extérieur (possible départ de mur ou contrefort) et un blocage constitué de blocs de lapis Gabinus et de tuf orangé mesurant entre 0,30 et $0,40 \mathrm{~m}$ de large pris dans un limon brun gris (MR 873).

Fig. 7. Vue aérienne zénithale du couloir C4.

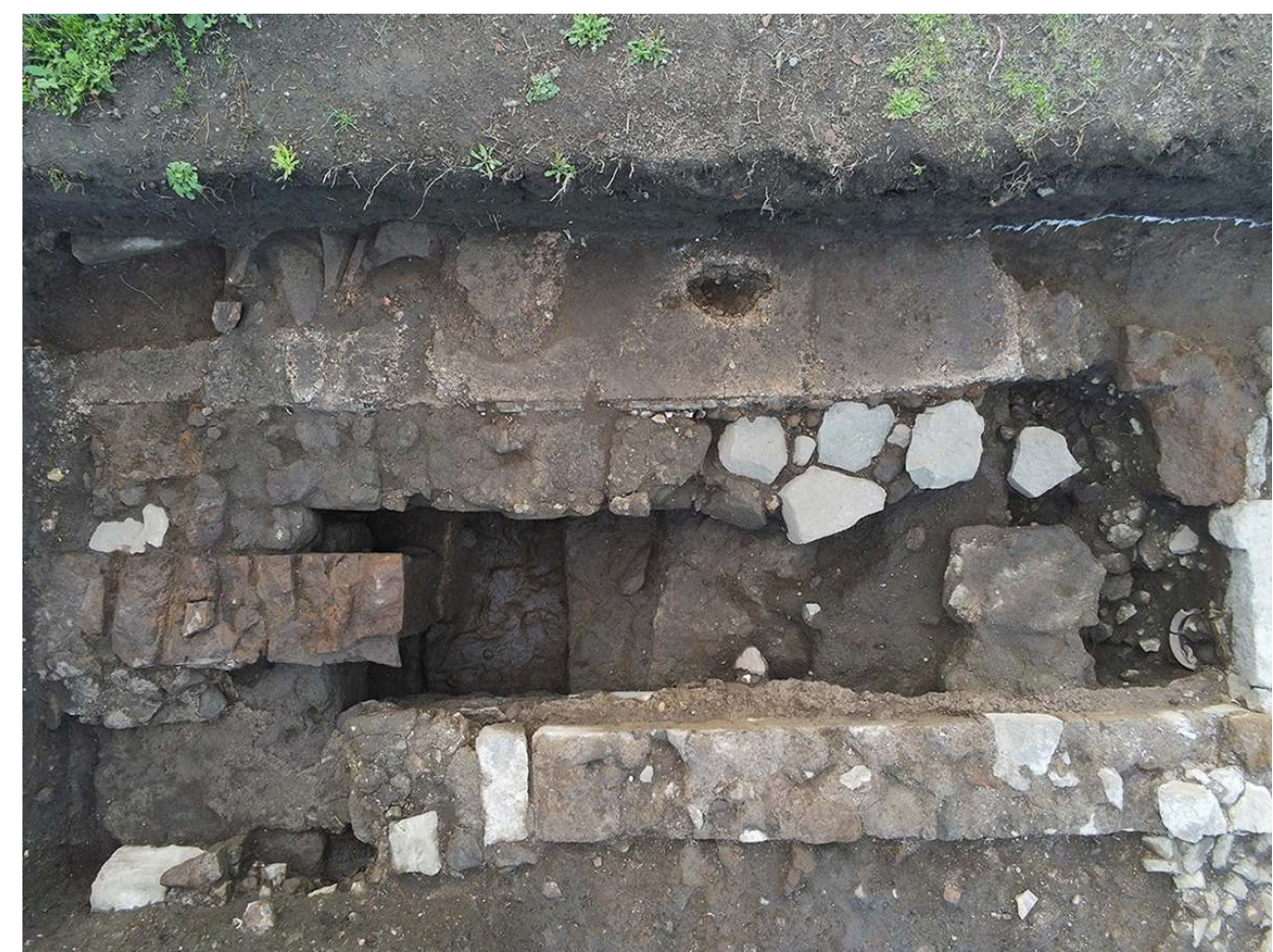

Mehdi Belarbi.

$\mathrm{Au}$ sud, l'extrémité nord d'un mur matérialisé par un bloc parallélépipédique de tuf orangé (MR 871) s'intercale entre les deux murs du couloir. Des maçonneries montées au mortier de chaux forment un angle droit avec ces derniers et s'adossent au mur MR 871, soit lié à un premier état de la domus, soit antérieur à la construction de celle-ci. À un endroit où l'on s'attendrait plutôt à trouver un passage, il se trouve en position perpendiculaire, dans l'axe du mur arrière du bâtiment (MR 114/95) qui clôt le tablinum et les pièces adjacentes au sud et au-delà duquel pourrait se trouver, selon le plan habituel pour ce type d'édifice, un hortus, voire un péristyle.

15 Cependant, le plan et le phasage architectural restent à préciser car la superficie ouverte est réduite et l'angle de mur situé à l'est de MR 871, celui formé par MR 72 et MR 903 et 904, a été récupéré, action matérialisée par la tranchée TR 894 (fig. 2).

Bulletin archéologique des Écoles françaises à l'étranger , Italie 
Le sol en béton de tuileau de l'atrium est moins dégradé dans la partie à l'est de l'impluvium (SO 675), il est constitué d'un mortier de chaux blanc beige contenant de très nombreux éclats $(0,5$ à $1 \mathrm{~cm})$ de terre cuite et de nombreux graviers (fig. 8). Sa surface est très détériorée et il ne subsiste plus de parties lisses. Des traces très localisées de peinture rouge ont néanmoins été perçues.

Fig. 8. Le sol SO 675.

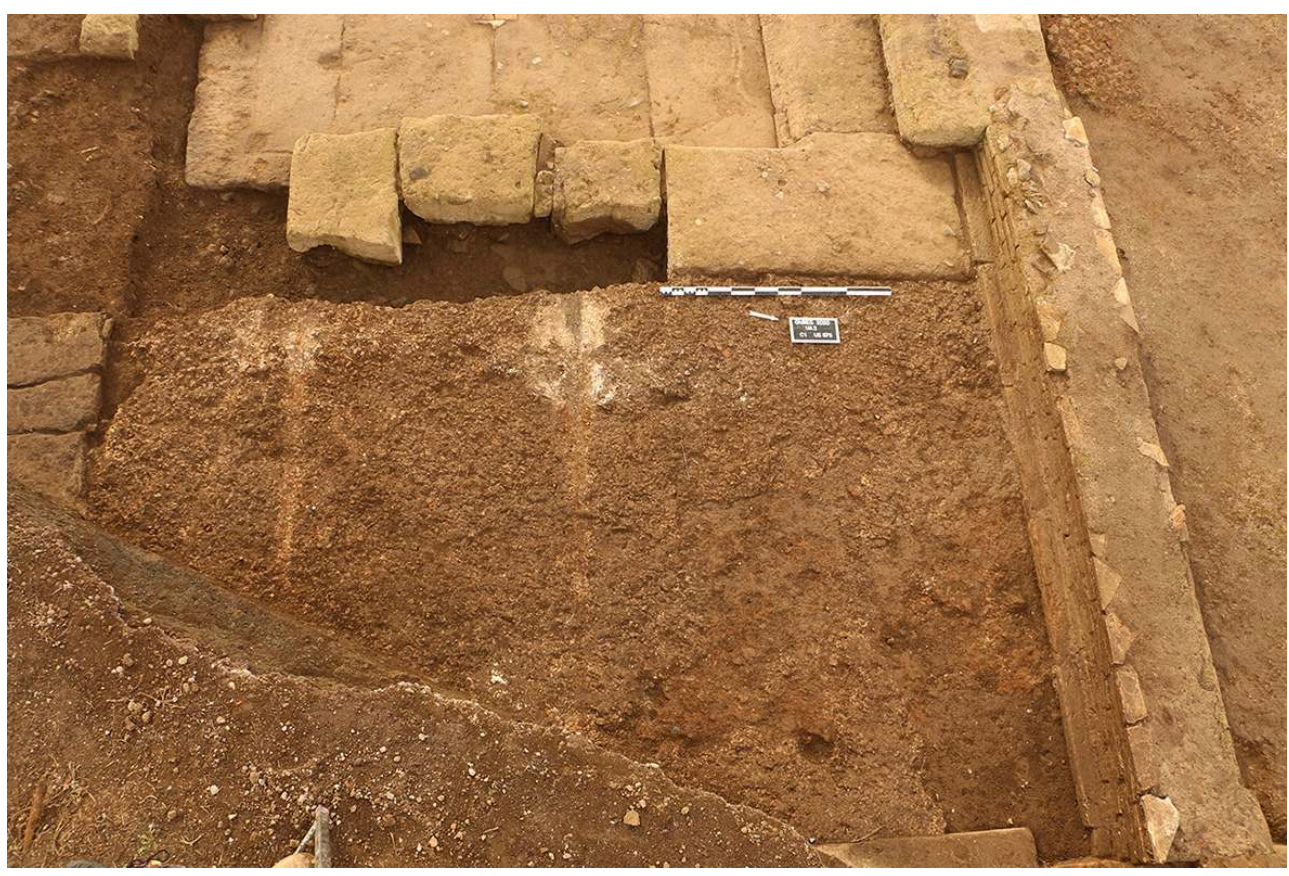

Johann Blanchard.

17 Une dalle de seuil a été partiellement dégagée au niveau de la berme orientale. Elle marque l'entrée d'une nouvelle pièce ( $\mathrm{C} 10)$ en position de symétrie avec la pièce $\mathrm{C} 2$ de l'autre côté de l'atrium à l'ouest. L'angle des murs qui séparent les pièces $\mathrm{C} 1, \mathrm{C} 10$ et $\mathrm{B} 9 \mathrm{a}$ également été mis au jour.

Une margelle de puits (PTS 879) a été découverte à l'angle sud-est de l'impluvium (fig. 9). Elle est construite en dalle rectangulaires de lapis Gabinus et son ouverture se place dans l'alignement d'un cuniculus (GA 755) taillé dans la paroi du puits 680 localisé à neuf mètres au nord, dans la pièce $\mathrm{B} 2$. 
Fig. 9. La margelle du puits 879.

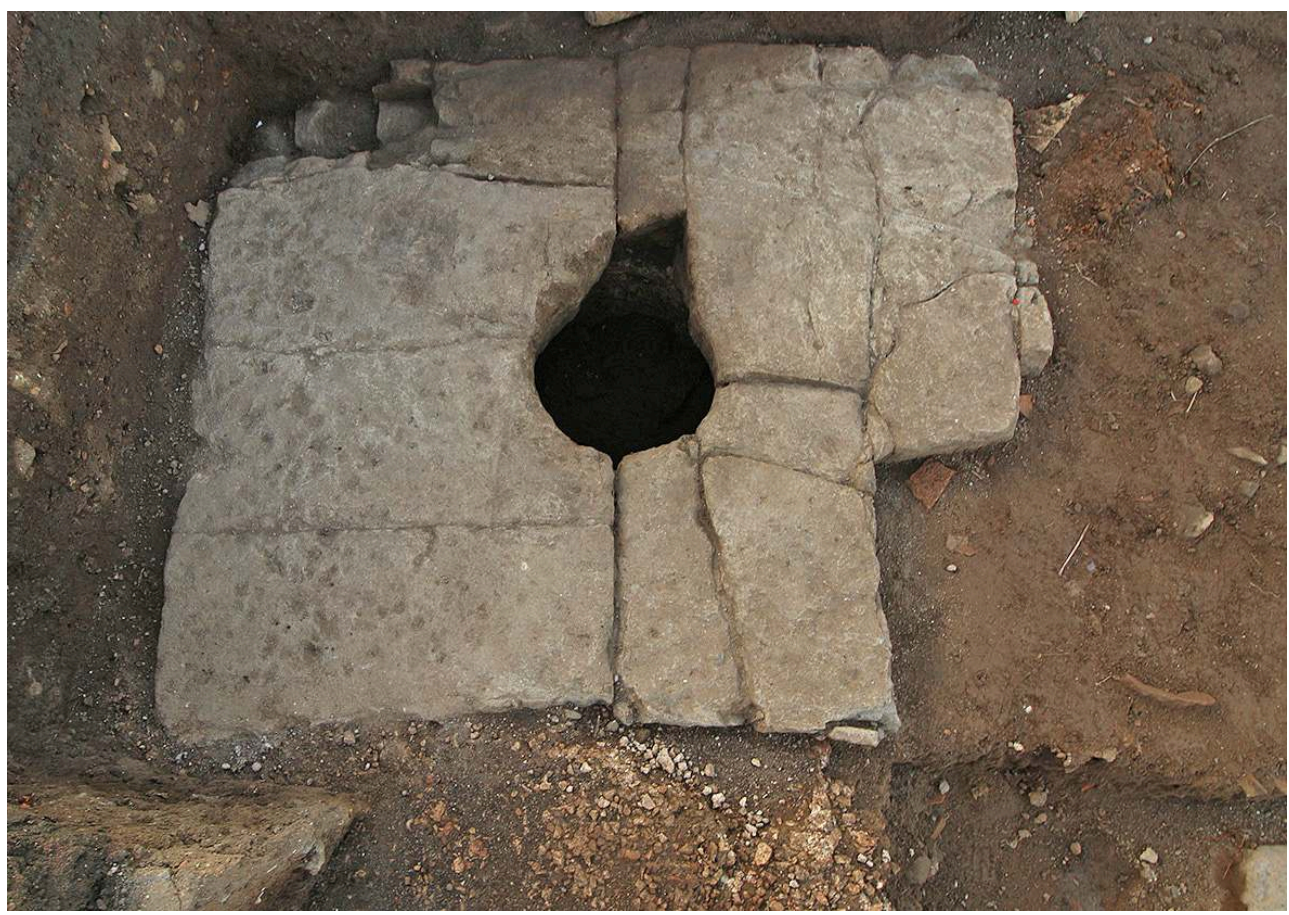

Mehdi Belarbi.

19 Le sol de la pièce B9, SO 676, est mieux conservé et s'apparente au sol SO 675. Sa surface présente un semis irrégulier de tesselles blanches (fig. 10). La pièce est scellée par un remblai de démolition qui a notamment livré une importante quantité d'ossements de faune, peut-être liés à une activité de boucherie.

Fig. 10. Le sol 676.

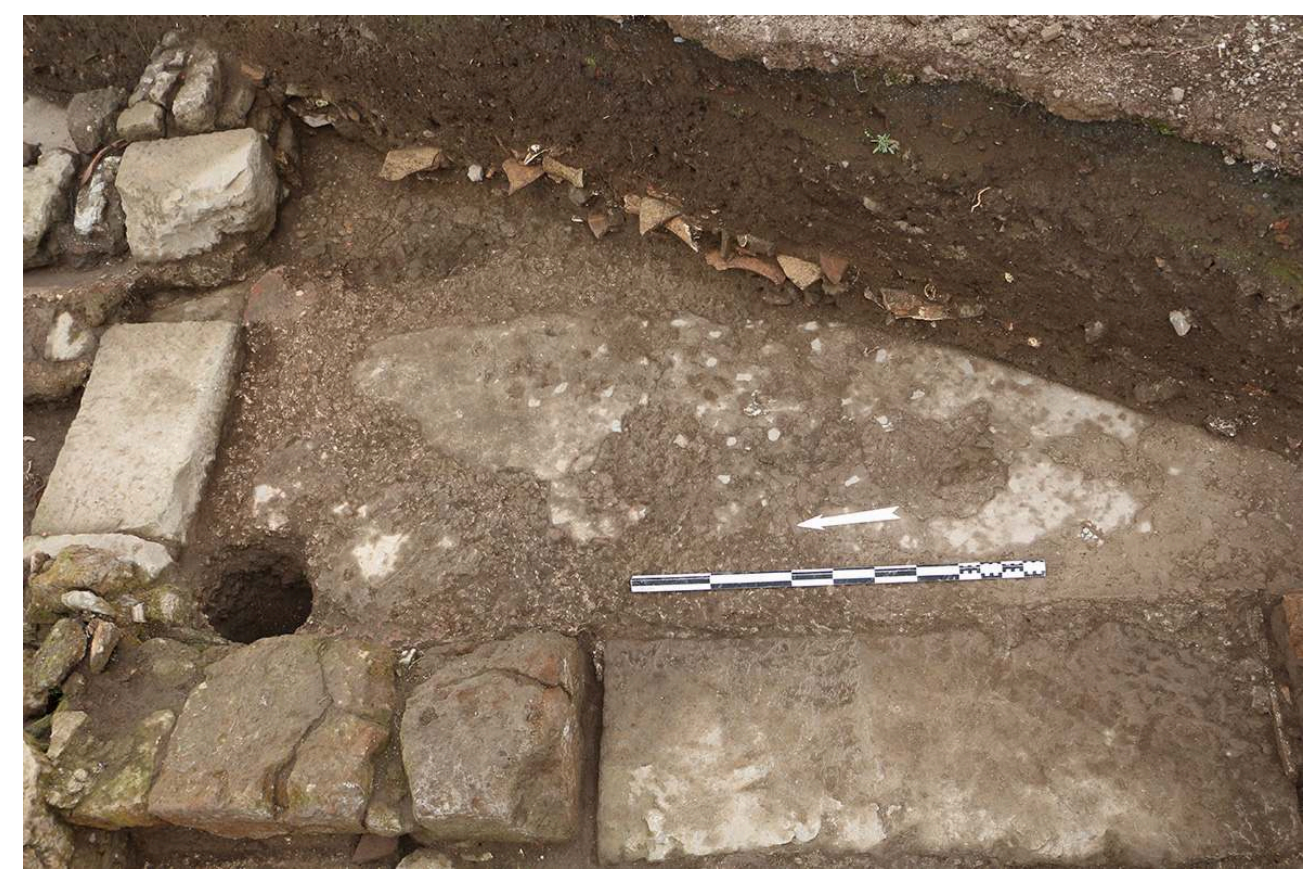

Johann Blanchard. 
La fouille de la pièce B8 a révélé qu'une galerie souterraine (GA 754) orientée nord-est/ sud-ouest creusée dans la paroi nord du puits 680 et se terminant peu avant le mur nord de la pièce sous la fosse FS 771, s'est effondrée entre la fin du $\mathrm{I}^{\mathrm{e}}$ et le premier tiers du II ${ }^{\mathrm{e}}$ s. apr. J.-C. (fig. 11 et 12). Ce puits est lui-même relié par une deuxième galerie nord/sud (GA 755) au puits PTS 879 alimenté par la citerne de l'impluvium ${ }^{4}$.

Fig. 11. Les installations hydrauliques de la pièce B8 avec une possible latrine au premier plan.

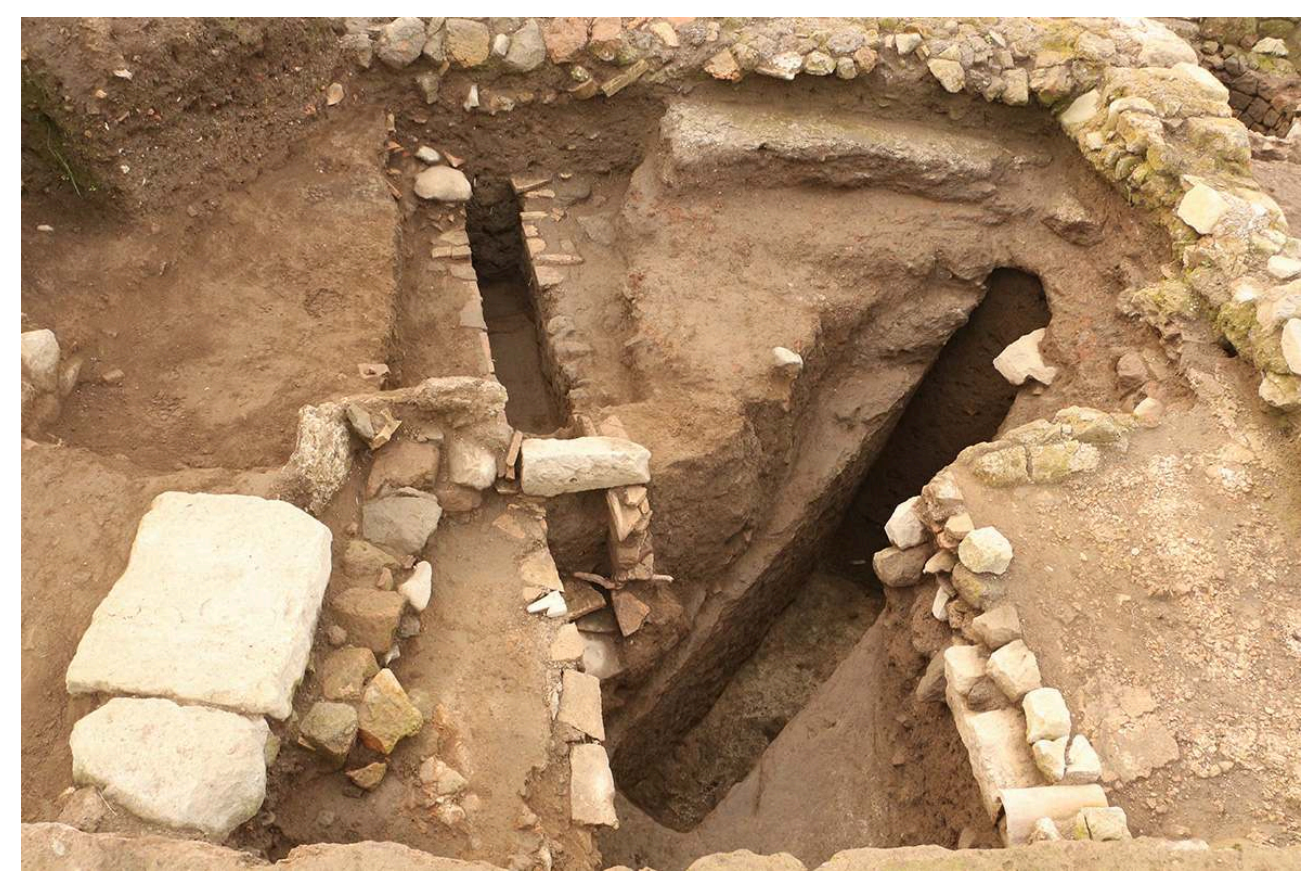

Heidelinde Hupfer. 
Fig. 12. Vue aérienne zénithale de l'atrium et du secteur est de la domus UA 2.

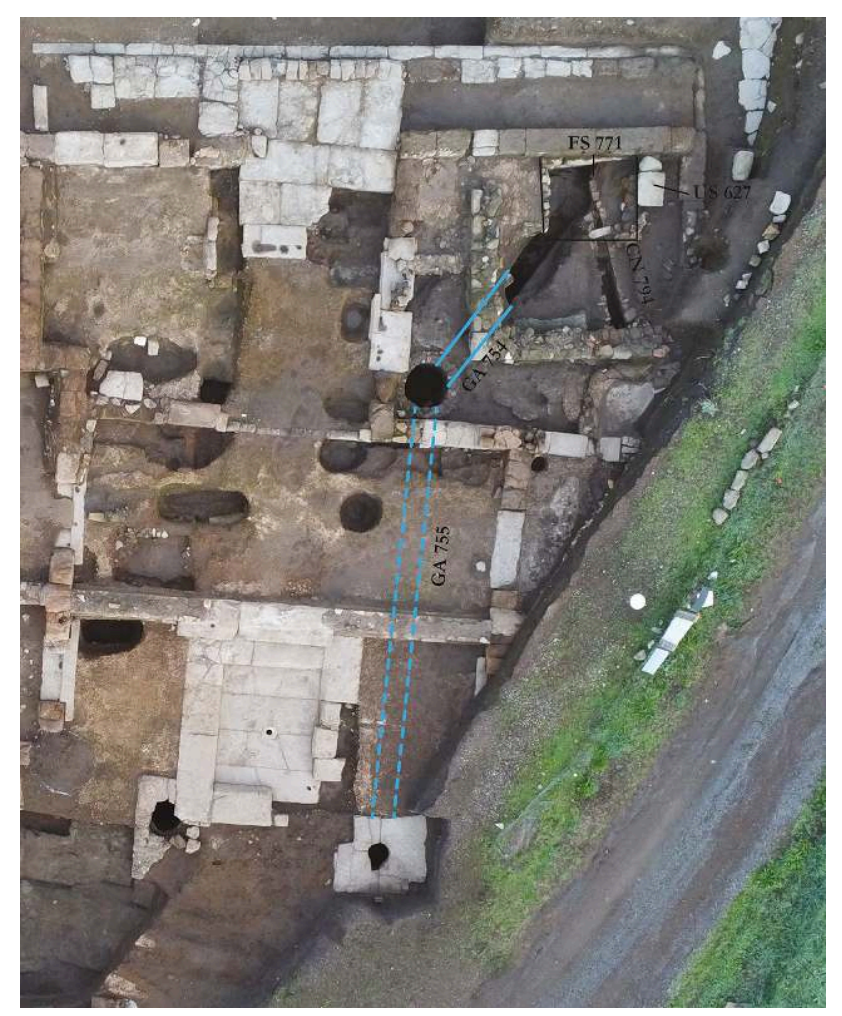

Mehdi Belarbi.

21 La fosse FS 771 s'appuie contre le mur nord de la pièce (MR 282, fig. 2). Elle est de forme rectangulaire et mesure 1,51 $\mathrm{m}$ de long sur 1,07 $\mathrm{m}$ de large pour une profondeur de $0,35 \mathrm{~m}$. Ses parois ouest et est sont construites avec des assises irrégulières de moellons et petits blocs de tufs ainsi que de fragments de terre cuite architecturale liés par un mortier de chaux pulvérulent (fig. 11 et 13). Une couche de mortier blanc pulvérulent qui recouvre la paroi sud de la fosse constitue le vestige d'une maçonnerie dont les éléments en pierre ne subsistent plus (à l'exception de rares moellons visibles en surface). Le remplissage, un limon sableux brun très riche en fragments de tuiles, a livré du mobilier céramique qui donne les années 80/90 apr. J.-C. comme terminus post quem pour l'abandon de la fosse. Dans la moitié orientale de la fosse, il est coiffé par les débris d'une chape de mortier blanc très altérée. Dans la moitié occidentale, située au-dessus de la galerie GA 754, une dalle de travertin a été exhumée en position effondrée. La canalisation $\mathrm{CN} 794$, considérée dans un premier temps comme antérieure à la fosse ${ }^{5}$, est en fait directement reliée à celle-ci et sa dalle de couverture est encore en place dans la paroi sud de FS 771. Ses parois sont construites avec des assises de tuiles plates remployées montées à la terre. Le fond est constitué de demi-briques bipédales recouvertes de concrétions blanchâtres dues à la circulation de l'eau et présente une pente à 9,8\% qui descend vers le sud-est en direction de la rue nord-sud VO 2. 
Fig. 13. La fosse FS 771 et les structures environnantes en 2019.

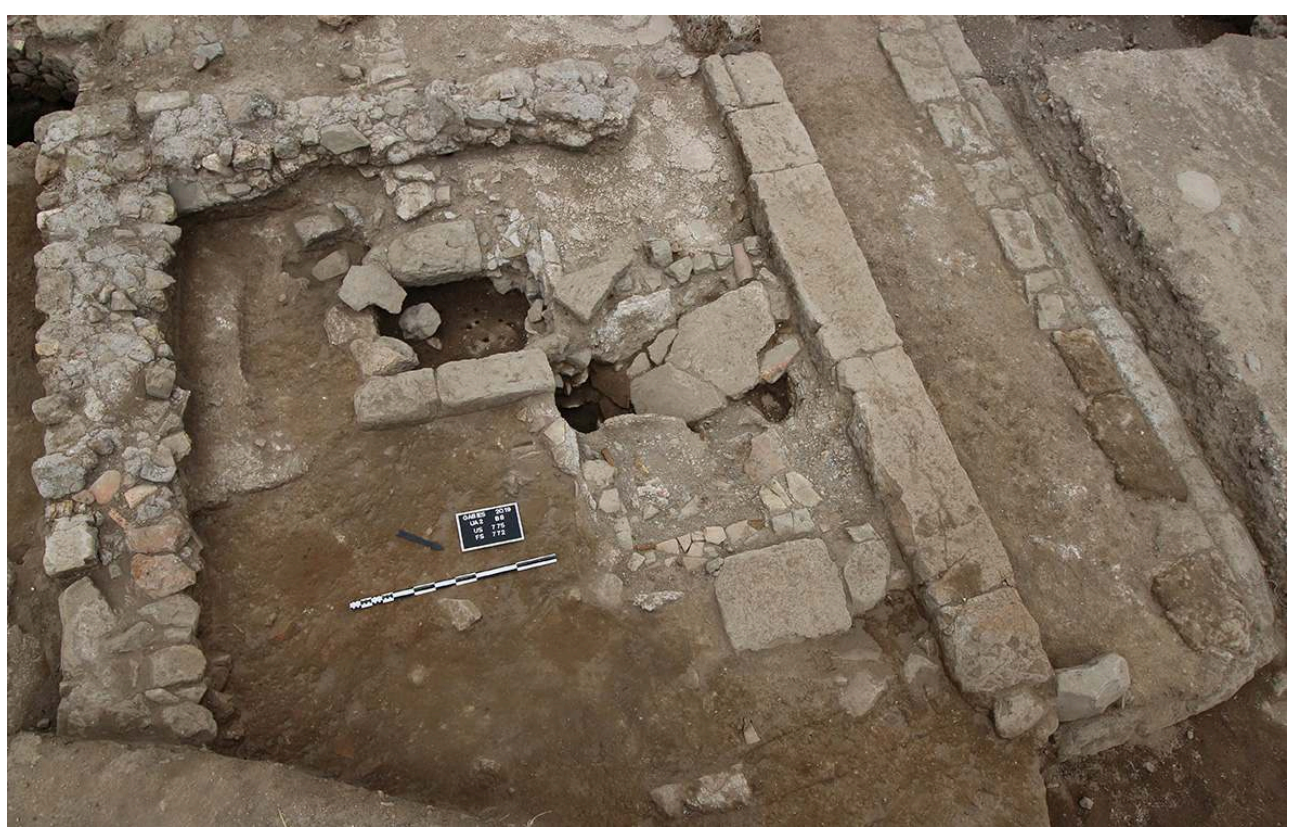

Pierre Broutin.

Cette canalisation faisait donc office d'exutoire pour les eaux usées qui étaient ainsi vraisemblablement rejetées dans un collecteur situé sous la rue. Ce dispositif et les aménagements hydrauliques voisins, le puits PTS 680 et le bassin BS 705 qui supposent un abondant usage de l'eau, conduisent à émettre l'hypothèse selon laquelle ce secteur de la domus se rapporte à un espace de cuisine et latrines. En effet, les exemples connus dans l'aire vésuvienne montrent que l'évacuation des eaux usées se faisait dans les latrines souvent situées à côté des cuisines ${ }^{6}$. Quand elles ne constituent pas une pièce à part, les petites latrines privées peuvent être séparées du reste de la cuisine par une cloison afin d'éviter d'être vu comme le rappelle Gemma Jansen ${ }^{7}$. On retrouve apparemment ce souci de discrétion dans la pièce B8 puisque les parois de la fosse FS 771 délimitent sans doute les cloisons d'une petite latrine à laquelle on accédait à l'est comme le montre la présence d'une petite dalle de tuf en lapis Gabinus (US 627, fig. 12 et 13) matérialisant un seuil.

La morphologie de cet aménagement rappelle, d'autre part, une structure similaire mise au jour dans la pièce L de la domus VI à Norba et interprétée comme une latrine ${ }^{8}$. La structure quadrangulaire SB 647 placée contre l'angle sud-ouest de la fosse FS $771^{9}$ peut de la même manière être comparée celle à mise au jour à proximité immédiate de la latrine de la pièce L de la domus VI de Norba. Cette dernière est circonscrite par des blocs de calcaires encadrant un niveau de briques en terre cuite disposées à plat sur une couche de terre argileuse et a été identifiée comme un foyer ${ }^{10}$. L'absence d'éléments en terre cuite en ce qui concerne la structure SB 647 s'explique du fait que la majeure partie de ce possible foyer a été détruite lors de l'effondrement de la galerie sous-jacente ${ }^{11}$.

24 La latrine et au moins une partie de la cuisine sont mises hors service suite à l'effondrement de la galerie GA 754. La pièce est ensuite divisée par un angle de murs en mortier qui délimite la zone effondrée. 
Une structure maçonnée quadrangulaire a été en partie mise au jour sous le bassin BS 705 à l'est de la pièce B2. Elle se présente sous la forme d'un radier en mortier de tuileau de couleur rose orangée contenant des inclusions millimétriques de terre cuite (fig. 14). Des fragments de tuiles jaunes sont disposés à plat en surface. Ses bords sont marqués par un léger ressaut (en terre cuite pour les parties bien conservées) de $2 \mathrm{~cm}$ de haut et $8 \mathrm{~cm}$ de large.

Fig. 14. La structure en mortier de tuileau US 909 sous le bassin BS 705.

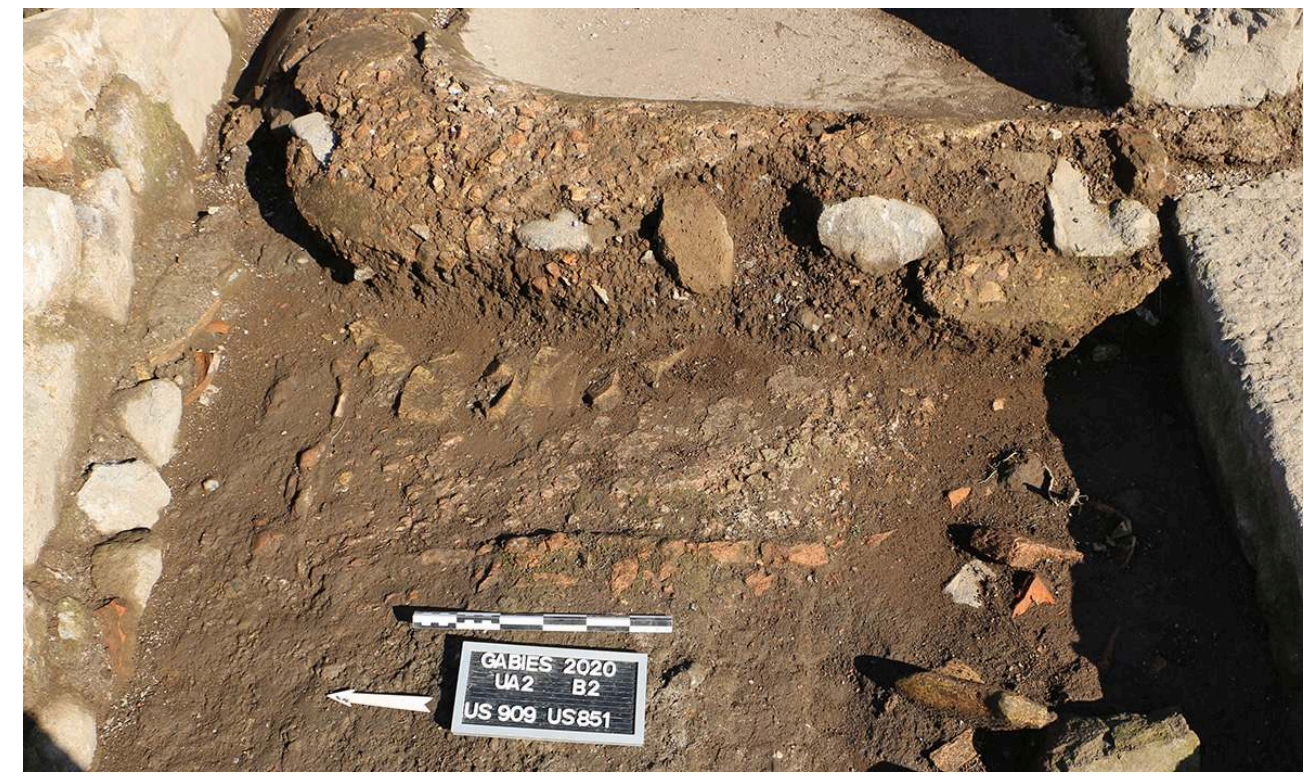

Laura Favreau.

La fouille de la pièce B7 a été étendue vers le sud et un mur monté au mortier de chaux a été retrouvé à l'ouest (MR 783). Il délimite ainsi une grande pièce de $4 \mathrm{~m}$ de large ouverte sur $7 \mathrm{~m}$ de long (fig. 15). Une plinthe d'enduit peint rouge est encore en place à la base du mur à la jonction avec le sol SO 758. Aucun mur de refend qui délimiterait la pièce au sud n'a pour l'instant été trouvé, ce qui confirme qu'il s'agit d'un grand espace dont la fonction reste à déterminer. 
Fig. 15. La pièce B7 et le mur MR 783 en partie récupéré au nord.

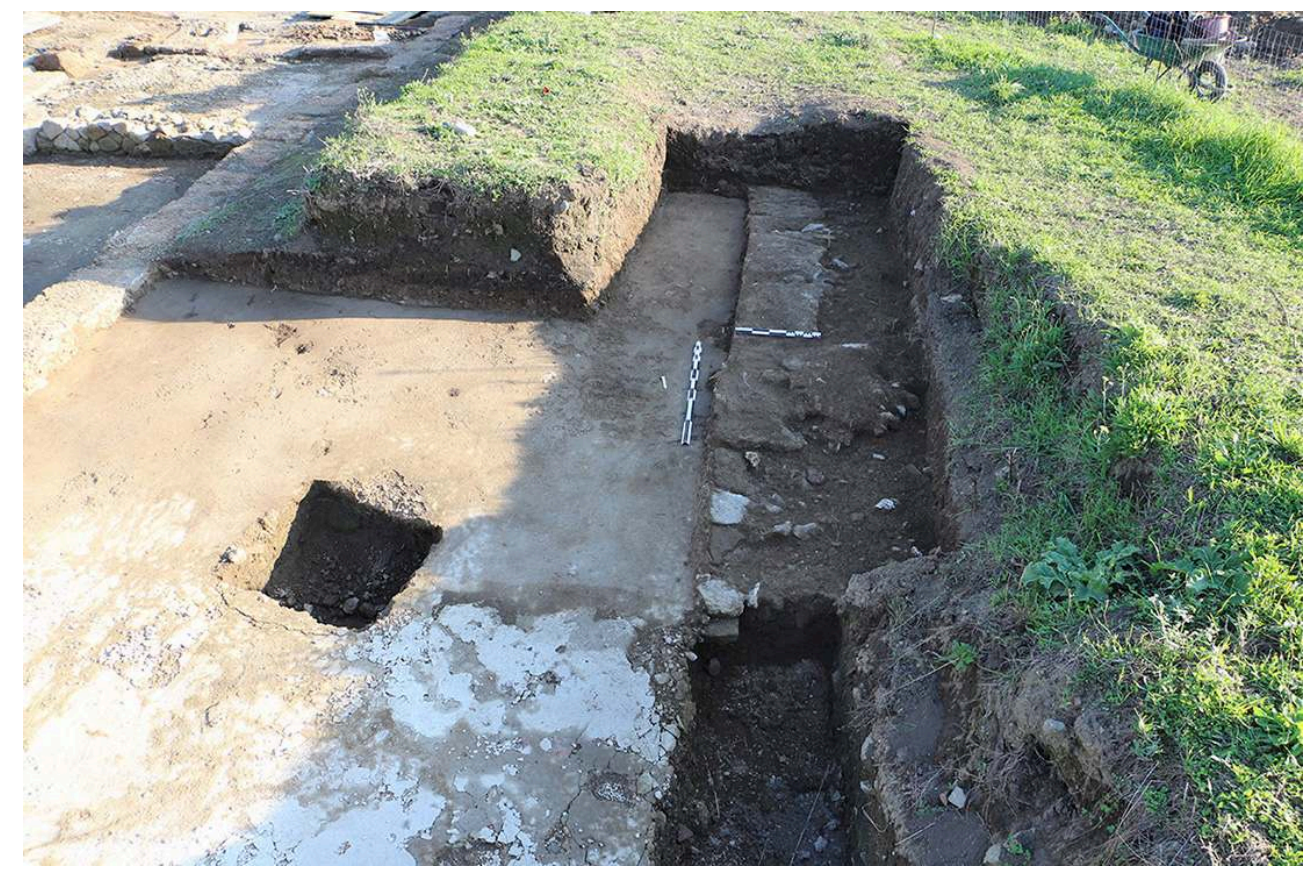

Marjolaine Benaich.

Le mur MR 783 se trouve sur le même alignement qu'un mur mis au jour lors de la première campagne de 2013, MR 98 (pièce C6). L'absence d'accès clairement identifier entre le long mur 453/70 implique que les pièces situées à l'ouest de celui-ci (dont B7 et B6 avant la construction du mur MR 454) appartiennent à un autre bâtiment mitoyen dont on devra déterminer à l'avenir s'il s'agit d'une autre domus ou non. En outre, le mur de façade septentrional, MR 840 adopte une orientation légèrement décalée vers l'ouest-nord-ouest par rapport au mur MR 270 ; le premier adopte une orientation de $109^{\circ}$ ouest par rapport au nord géographique et le second une orientation de $111^{\circ}$ ouest. Ce changement d'orientation correspond au tracé originel curviligne de VO 1 qui suivait la pente du cratère avant la construction du sanctuaire de Junon Gabina.

\section{L'angle sud-est du sanctuaire : le secteur du théâtre}

28 Un troisième pilier de fondation, PI 831, a été mis au jour dans le caisson A3 dans l'alignement de ceux exhumés auparavant dans les caissons A1 et A2 (PI 249 et 383). Comme les deux autres, il s'agit d'un bloc de lapis Gabinus parallélépipédique de 0,60 m de large posé de chant dont seul le lit d'attente a été dégagé (fig. 16). 
Fig. 16. Le pilier de fondation PI 831 dans le caisson A3.

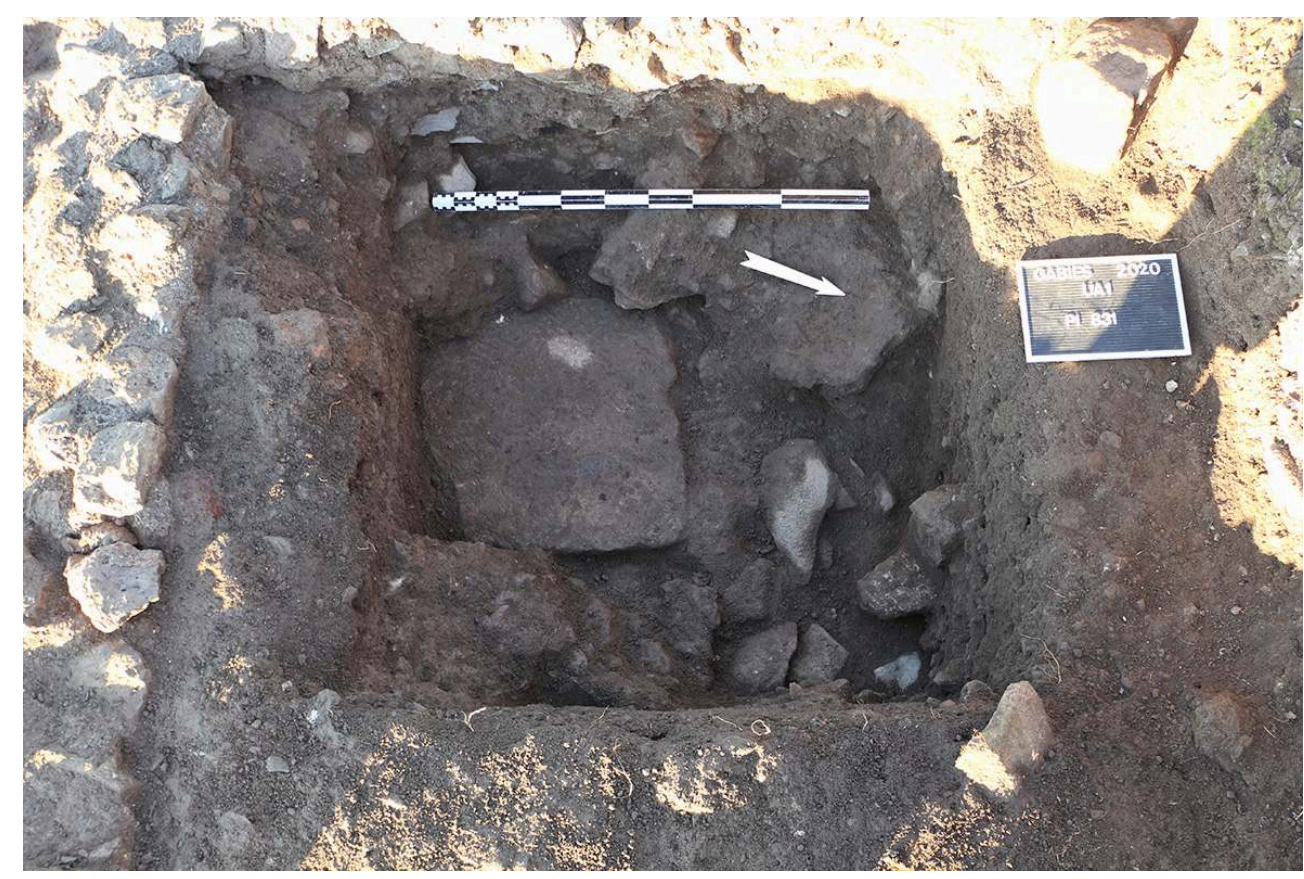

Priscillia Debouige.

Dans l'espace A5, une deuxième pile de fondation (PI 920) en opus caementicium est accolée au mur de fondation de la frons scaenae, MR 559 (fig. 17). Son entraxe avec PI 465 est de 3,40 m, tout comme l'entraxe entre PI 465 et PI 464 est de 3,40 m également. Ces trois piles supportaient des piédestaux de colonne.

Fig. 17. L'espace A5 - campagne de 2020.

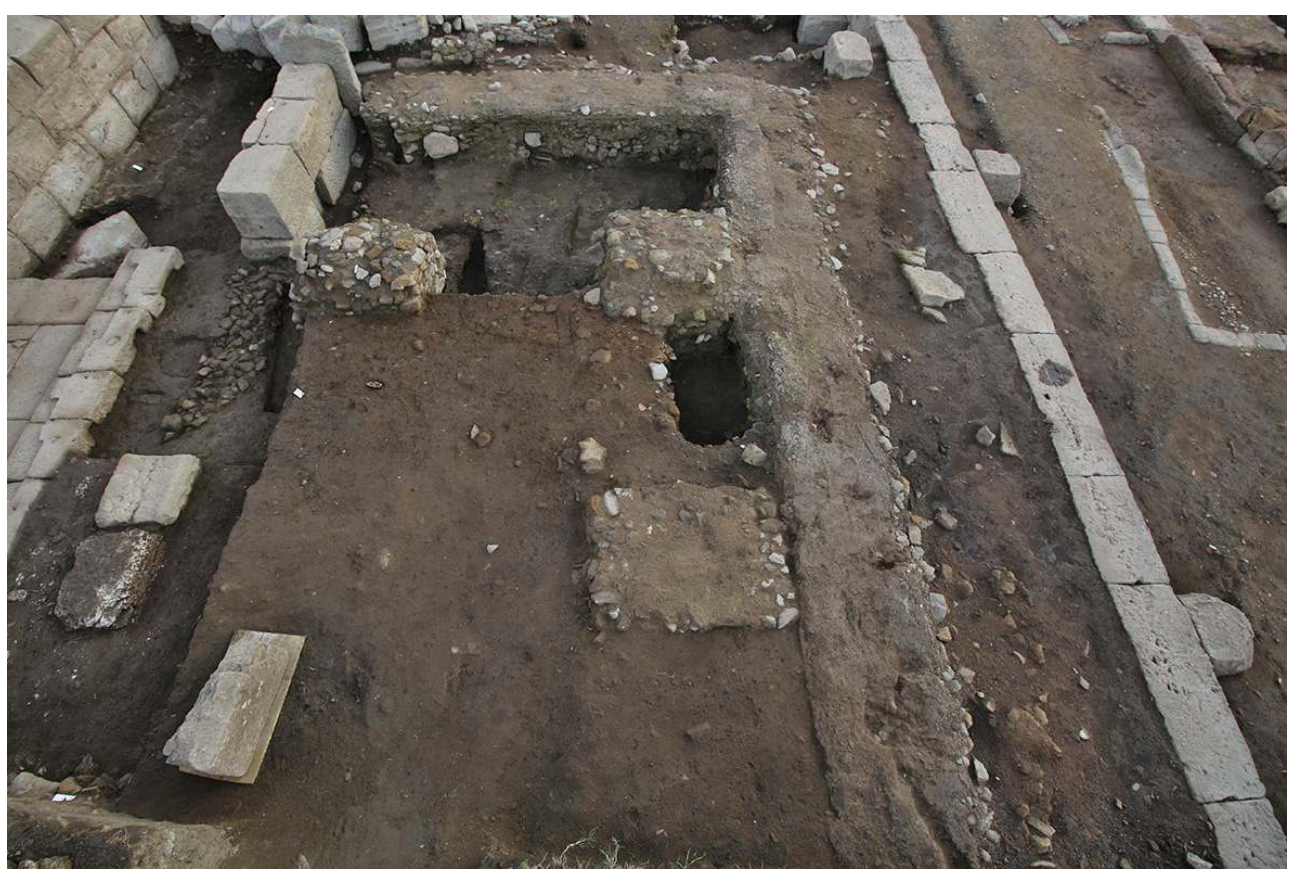

Mehdi Belarbi. 
Dans l'espace A6, à l'ouest de l'aditus fouillé en 2019, la première assise de la frons pulpiti (MR 889) a été découverte sur un peu plus de $5 \mathrm{~m}$ de long (fig. 18). Il s'agit d'un appareil rectangulaire de blocs en lapis gabinus disposés en panneresse, d'une vingtaine de centimètres d'épaisseur, dont la largeur moyenne est de 0,64 $\mathrm{m}$ et dont la longueur varie entre 0,56 et $1,26 \mathrm{~m}$. Ils sont moulurés pour accueillir la décoration du mur d'estrade. Des encoches situées sur la face aveugle suggèrent qu'il pourrait s'agir de blocs de remploi et elles indiquent qu'ils ont été déplacés. Des empreintes de goujons sont encore perceptibles et la surface destinée à accueillir l'enduit est seulement démaigrie afin de faciliter l'accroche.

Fig. 18. L'aditus maximus oriental et le passage entre la scène et le mur de soutènement des gradins vers l'orchestra.

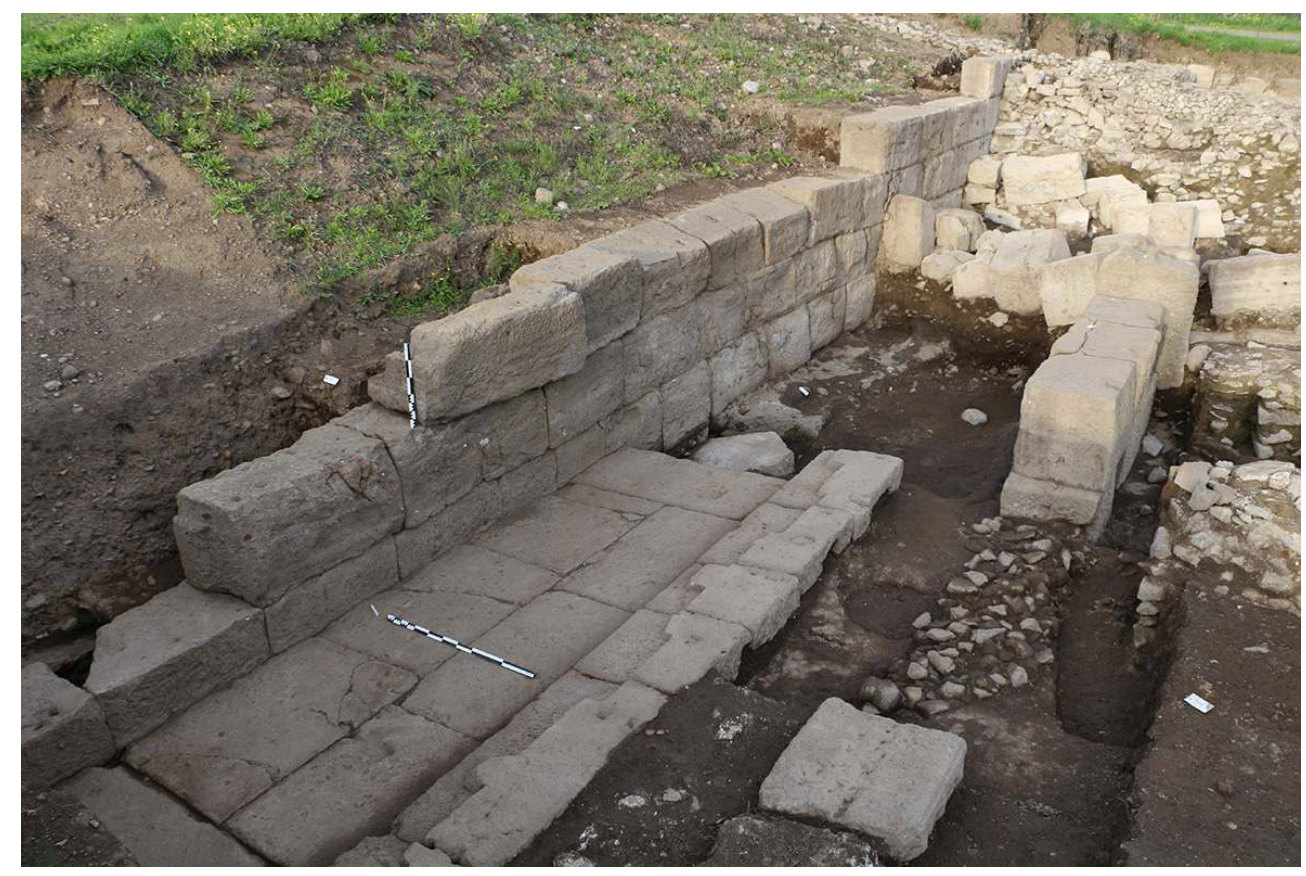

Antoine Valois.

Deux blocs d'élévation sculptés qui ornaient ce même mur ont été retrouvés effondrés dans le passage latéral. Ces éléments montrent que ce mur était rythmé par des niches rectangulaires à décor géométrique scandé de colonnettes mesurant $0,18 \mathrm{~m}$ de diamètre (fig. 19). Des traces ténues d'enduit à la chaux sont encore visibles sur certaines parties des blocs et témoignent que le mur recevait également un décor de stuc. 
Fig. 19. Bloc mouluré de la frons pulpiti repositionné sur la base du mur MR 889.

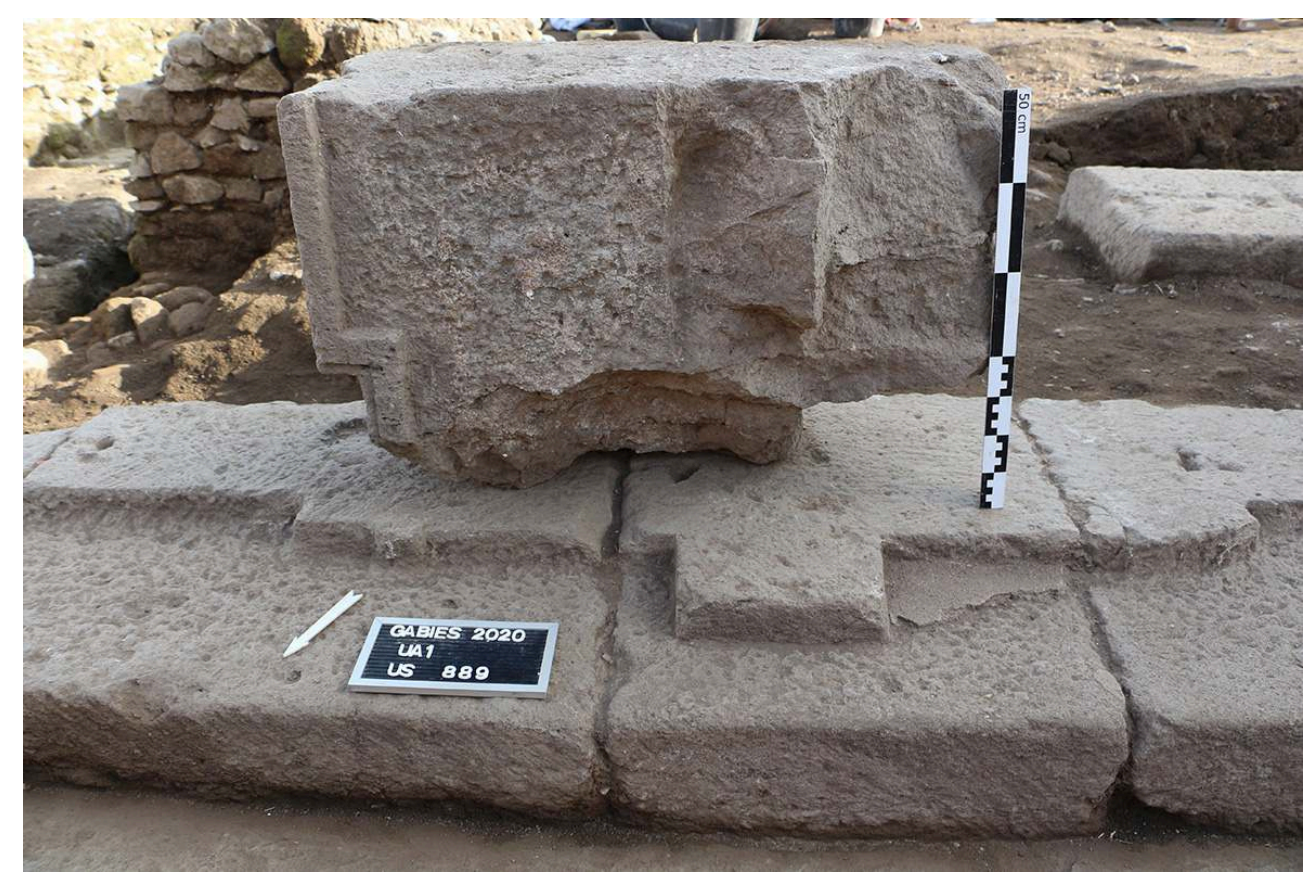

Mehdi Belarbi.

32 Entre la frons pulpiti (MR 889) et le mur de soutènement des gradins (MR 604), le dallage (SO 910) est bien conservé, contrairement au passage voûté de l'aditus où l'ancien sol a été intégralement récupéré. Deux dalles disposées perpendiculairement à ces murs sur toute la largeur du sol et espacées de 3,50 m encadrent deux rangées accolées de trois dalles parallèles. La rangée nord mesure $0,78 \mathrm{~m}$ de large et la rangée sud $0,70 \mathrm{~m}$ de large.

33 La frons pulpiti ne se place pas dans l'alignement du piédroit sud du passage voûté de l'aditus (MR 463) mais en position avancée de 0,67 m, réduisant ainsi le passage vers l'orchestra. En effet, il s'agit d'un deuxième état de construction du pulpitum au cours duquel celui-ci a été agrandi, vraisemblablement durant l'époque julio-claudienne. Selon toute hypothèse, le mur d'estrade n'a pas été reconstruit mais plutôt démonté et replacé un peu plus en avant.

\section{Conclusion}

34 La campagne de 2020 a permis de préciser le plan et la chronologie la domus UA 2 et d'établir qu'elle a été construite vers le milieu du $\mathrm{III}^{\mathrm{e}} \mathrm{s}$. av. J.-C. soit un siècle avant la monumentalisation du sanctuaire de Junon Gabina. Il est désormais possible de restituer un édifice de $430 \mathrm{~m}^{2}$ pour le secteur d'habitation et de $670 \mathrm{~m}^{2}$ dans l'hypothèse d'un jardin (voire d'un péristyle sur l'arrière) ${ }^{12}$.

35 Dans le secteur du théâtre, la découverte d'un troisième pilier de fondation dans le caisson A3 confirme l'existence d'une colonnade et, par extension, d'un portique qui bordait le côté est de l'édifice. À l'ouest, le très bon état de conservation du dallage qui borde la frons pulpiti, laisse présager que le sol de l'orchestra est vraisemblablement de même nature et tout aussi bien préservé. Notre connaissance de l'architecture du théâtre et de son évolution est désormais mieux connue avec une frons scaenae 
rectiligne dotée d'une columnatio, une frons pulpiti ornée de niches et de colonnettes, et des espaces extérieurs dallés en lapis Gabinus comme c'est généralement le cas à Gabies.

Sous les vestiges bâtis d'époque républicaine commencent à émerger des niveaux d'occupation et une nécropole d'époque archaïque mais, étant donné les faibles superficies dégagées pour la période, aucun vestige structuré de l'habitat antérieur au III ${ }^{\mathrm{e}}$ s. av. J.-C. n'est pour l'instant apparu dans ce secteur.

\section{BIBLIOGRAPHIE}

Almagro-Gorbea 1982

M. Almagro-Gorbea (dir.), El Santuario de Juno en Gabii: excavaciones 1956-1969, Rome, Bibliotheca italica $17,1982$.

Evans 2018

J.M. Evans, « Archaic Burials from Gabii, Italy », dans BABESCH 93, 2018, p. 27-48.

Glisoni - Hasselin - Roger 2018

S. Glisoni, I. Hasselin, D. Roger, « Gabies. Campagne 2017 du musée du Louvre et de la Surintendance de Rome ", dans Chronique des activités archéologiques de l'École française de Rome, 2018 (http://journals.openedition.org/cefr/1905, consulté le 03/09/2019).

Glisoni - Hasselin-Rous - Roger 2017a

S. Glisoni, I. Hasselin-Rous, D. Roger, « Gabies », dans Chronique des activités archéologiques de l'École française de Rome, 2017 (http://cefr.revues.org/1644, consulté le 06/12/2017).

Glisoni - Hasselin-Rous - Roger 2017b

S. Glisoni, I. Hasselin-Rous, D. Roger, « Gabies. Campagnes 2014 et 2016 du Musée du Louvre et de la Surintendance de Rome ", dans Chronique des activités archéologiques de l'École française de Rome, 2017 (http://journals.openedition.org/cefr/1644, consulté le 03/09/2019).

Glisoni - Zanella 2019

S. Glisoni - S. Zanella, « Gabies. Campagne de 2018 du musée du Louvre et de la Surintendance de Rome ", dans Chronique des activités archéologiques de l'École française de Rome, 2019 (http:// journals.openedition.org/cefr/3980, consulté le 16/12/2019).

Jansen 1997

G. Jansen, « Private Toilets at Pompeii: Appearance and Operation », dans S.E. Bon, R. Jones (dir.), Sequence and space in Pompeii, Oxford, Oxbow monograph 77, 1997 p. 121-134.

Jansen 1991

G.C.M. Jansen, « Water systems and Sanitation in the houses of Herculaneum », dans Mededelingen van het Nederlands Historisch Instituut te Rome - Antiquity 50, 1991, p. 145-166.

Johnston et al. 2018

A.C. Johnston, M. Mogetta, L. Banducci, R. Opitz, A. Gallone, J. Farr, E.C. Cicci, N. Terrenato, « A monumental mid-republican building complex at gabii », dans Papers of the British School at Rome 86, 2018, p. 1-35. 
Opitz - Mogetta - Terrenato 2016

R. Opitz, M. Mogetta, N. Terrenato, A Mid-Republican House From Gabii, 2016.

Quilici - Quilici Gigli 2014

L. Quilici, S. Quilici Gigli (dir.), Norba: domus e materiali, Roma, Atlante tematico di topografia

antica Supplemento 19, 2014.

\section{NOTES}

1. Il s'agit de deux US appartenant à un même niveau lacunaire ; elles sont séparées par une remontée du banc de tuf.

\section{Evans 2018.}

3. Glisoni - Hasselin - Roger 2018.

4. Un premier puits (PTS 27) a été mis au jour contre l'angle sud-est du bassin de l'impluvium. La découverte du puits PTS 879 lors de la campagne de 2020 et le fait que l'angle du bassin déborde légèrement sur la bouche du puits PTS 27 montrent que ce dernier appartient à un état antérieur, avant l'installation du bassin BS 18. Sur l'atrium et l'impluvium cf. Glisoni - Zanella 2019 ; sur le puits PTS 680 cf. Glisoni 2020.

5. Glisoni 2020, p. 8.

6. Jansen 1991 et Jansen 1997.

7. Jansen 1997, p. 125-126.

8. Quilici - Quilici Gigli 2014, p. 32-33.

9. Afin de ne pas entraîner de confusion, le terme de fosse est utilisé ici dans une acceptation neutre issue du terme dont a été qualifiée cette structure lors de la fouille et ne fait pas référence à une fosse d'aisance qui aurait été régulièrement vidangée à la main puisque la canalisation $\mathrm{CN} 794$ implique un rinçage très régulier de la latrine.

10. Quilici - Quilici Gigli 2014, p. 50.

11. La position de SB 647 au-dessus de cette galerie a dans un premier temps conduit à émettre l'hypothèse d'un puits. Cependant, la présence du puits PTS 680, très proche, son orientation divergente de celle de la galerie et le fait que les deux blocs de tuf parallélépipédiques encore en place reposent directement sur un niveau de terre brune et ne constituent donc pas la partie haute d'un cuvelage, sont autant d'éléments invitant à écarter cette hypothèse, celle d'un foyer semblant, par comparaison avec la découverte de Norba, plus pertinente.

12. La limite sud étant hypothétiquement placée au milieu de l'insula dans laquelle s'insère la domus UA 2. 


\section{INDEX}

sujets https://ark.frantiq.fr/ark:/26678/pcrtF9P4mbuyGk, https://ark.frantiq.fr/ark:/26678/ crtbBZ4OXcUbq, https://ark.frantiq.fr/ark:/26678/pcrt68T1KcWNtb, https://ark.frantiq.fr/ark:/ 26678/pcrtBTWy6SwZAu, https://ark.frantiq.fr/ark:/26678/pcrtcnLd0ZlR3X, https:// ark.frantiq.fr/ark:/26678/pcrt795b632nWw

Année de l'opération : 2020

chronologie https://ark.frantiq.fr/ark:/26678/pcrtB2qQmSnkAM, https://ark.frantiq.fr/ark:/ 26678/pcrtoNSSGgIkbX, https://ark.frantiq.fr/ark:/26678/pcrtH5r3FYBpwe

Thèmes : EFR

lieux https://ark.frantiq.fr/ark:/26678/pcrtawfW2EpsFF

\section{AUTEURS}

\section{STEVE GLISONI}

Département des antiquités grecques, étrusques et romaines du Musée du Louvre - INRAP, UMR 7041 ArScan 